\title{
Link Suggestions in Terrorists Networks Using Semi Discrete Decomposition
}

\author{
Vaclav Snasel*, Zdenek Horak* and Ajith Abraham ${ }^{\dagger}$ \\ ${ }^{*} V \check{S} B-T e c h n i c a l$ University of Ostrava, 17. listopadu 15 \\ 70833 Ostrava, Czech Republic \\ Email:vaclav.snasel@vsb.cz, zdenek.horak.st4@vsb.cz \\ $\dagger$ Machine Intelligence Research Labs (MIR Labs), \\ Scientific Network for Innovation and Research Excellence, USA \\ Email: ajith.abraham@ieee.org
}

\begin{abstract}
Recently several terrorist acts have created disruptions in the airline industries, tourism as well as the financial markets. It is believed just like the World War II had accelerated the development of nuclear energy, the longerterm impact of the current war on terrorism could lead to the allocation of major resources (manpower, funding etc.) towards dealing with this. In this paper, we present a social network concept to visualize a terrorist network. Visualization is very important part for analyzing a network since it can quickly provide good insight into the network structure, major members, and their properties. We used a matrix factorization methods called Semi Discrete Decomposition, which is highly suitable for dealing with huge networks. Empirical results using the 9-11 network data illustrate the efficiency of the proposed approach.
\end{abstract}

Keywords-Terrorism, network, visualization, semi discrete decomposition

\section{INTRODUCTION}

Terrorism deals with violent acts aiming to simulate fear, coercion, or intimidation [11]-[13]. It is an established fact that terrorism poses both direct and indirect threats to normal life. Even though terrorist strikes destroy only a small fraction of the direct economy of a country, their large effect on economic outcomes are well known [18] . It is sad to note that technical development are not only pulled by the demand for hi-tech products but is also highly influenced by some external environments, for example, terrorism. As shown by the aftermath of the 9/11 attacks, Bali, Madrid and London bombings, some changes have occurred in the day-to-day living of citizens of those countries [14]-[17].

After the 9/11 attacks, lots of efforts have been done to develop effective methods for anti-terrorism strategies. Our approach is focused on using social network concepts to visualize terrorist networks. Visualization is very important part for analyzing a network since it can quickly provide good insight into the network structure, major members, and their properties [4]. Analyzing huge networks is not an easy task and there is a need to reduce the complexity of these networks, which is usually depicted in the form of huge matrices. Matrix factorization method is a well established approach and in this research we used a Semi Discrete Decomposition, which is highly suitable for dealing with huge networks. Empirical results using the 9-11 network data illustrate the efficiency of the proposed approach.

The analysis of general complex networks is welldescribed in [1] and [2]. Our research is closely related to the link prediction, which is well elaborated by LibenNowell and Kleinberg [10]. Koren et al. [7] discussed the usage of matrix factorization methods for recommendation systems. This paper is based on well-known paper by Krebs [8], which is further discussed in [3].

Rest of the paper is organized as follows: In the following Section, Matrix factorization methods are introduced. Experimental details are provided in Section 3 followed by some Conclusions.

\section{MATRIX REDUCTION METHODS}

The matrix factorization methods are heavily used in many different fields. Roughly speaking, their principle lies in the decomposition of one matrix into several smaller matrices. After multiplying these matrices back, we get the same (or almost the same) matrix as the original one. The main advantage lies in uncovering hidden structures and relations in the data, which are later used to represent original information (or the most important parts of it) using much smaller space.

There are several matrix factorization methods, each suitable for different purpose. Singular Value Decomposition (see [5]) has been proved to be the best factorization under certain conditions. However, there are several drawbacks of this general method. Non-negative matrix factorization (see [9]) uses constraints producing non-negative basis vectors. This allows meaningful interpretation of factorization results in many different fields.

\section{A. Semidiscrete Decomposition (SDD)}

Semidiscrete Decomposition is specially designed to work on large binary data (the relation between objects exists or not). The advantages are clear - small memory consumption, fast computation and natural and direct interpretation of results without any additional rounding or thresholding. This method is therefore well-suited for applications in Latent Semantic Indexing. Technically speaking, for the original 


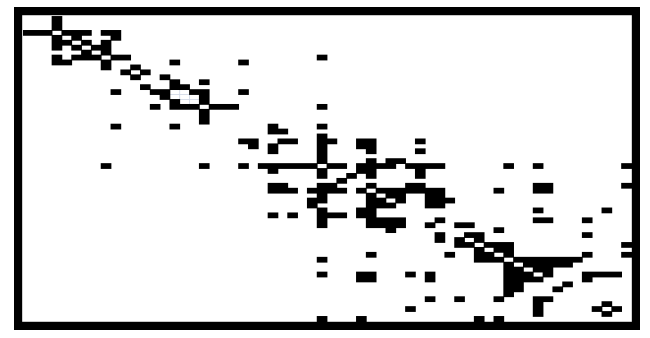

Figure 1. Original data matrix

data matrix $A$ we can compute reduced k-rank approximation $A_{k}=X_{k} D_{k} Y_{k}^{T}$, where $k$ is much smaller than the original dimension of $A$. For detailed explanation of SDD please see Kolda and O'Leary [6]. Most of the mentioned methods allow additional partial updates, so we do not have to compute the whole factorization from scratch.

\section{EXPERIMENTAL SETUP AND RESUlts}

Our experiment is based on the dataset involving 9/11 attacks from [8]. We have created a binary incidence matrix of involved persons as illustrated in Figure 1. Then we computed the $k$ rank SDD factorization and compared the reduced matrix with the original one. Change from zero to one in the reduced matrix can be in wider sense considered as a link suggestion. In different fields the suggestion can have different meanings. In the terrorist network, we can consider them as a suggestions to investigate, whether the link truly exists in reality.

The results for parameter setting $k=10$ are illustrated in Figure 2. Same coloring is used as in the original paper [8] by Krebs. Green triangles for flight AA \#11, which crashed into the WTC North, full red squares for flight AA \#77 which crashed into Pentagon, empty blue squares for flight UA \#93 which crashed into Pennsylvania and full purple diamonds for flight UA \#175 which crashed into WTC South.

Edges drawn in bold red are suggestions obtained by the mentioned reduction. We can see the suggested links in the group of Zacarias Moussaoui, Abu Qatada, David Courtaillier, Jerome Courtaillier, Abu Walid, Kamel Daoudi and Djamal Beghal. This group is also connected using several subgroups in the original data, therefore the proposed method suggests their stronger interconnection. The same holds for the suggested link between Ramzi bin al-Shibh and Lofti Raissi as it connects two different groups of individuals. Remaining suggestions can be explored in a similar way.

Results obtained using parameter setting $k=20$ (that means lower ratio of reduction) are shown in the right part of Figure 3. Less reduction in this case means less suggestions, but the suggestion obtained for rank 20 is not subset of suggestions for rank 10. As SDD always tries to minimize the error function, the reduction process is not straightforward - for example the links between Mehdi Khammoun and Zacarias Moussaoui, Mustafa Ahmed alHisawi and Satam Suqami as well as the link between Marwan Al-Shehhi and Nawaf Alhazmi are present at rank 20, but disapper at rank 10. The remaining links are still present at rank 10. Similar situation is with the setting $k=5$ (left part of Figure 3), which gives us 16 suggestions - using stronger reduction we have obtained more suggestions, but not all suggestions from rank 10 are present.

\section{CONCLUSIONS}

We presented a social network concept to visualize terrorist networks. We used the Semi Discrete Decomposition method, which is highly suitable for dealing with huge networks. Visualization results using the 9-11 network data illustrate the efficiency of the proposed approach.

\section{REFERENCES}

[1] S. Boccaletti, V. Latora, Y. Moreno, M. Chavez and D.U. Hwang: Complex networks: Structure and dynamics, Physics Reports, vol. 424, pp. 175-308, 2006

[2] L.F. Costa, FA Rodrigues, G. Travieso and P.R.V. Boas: Characterization of complex networks: A survey of measurements, Advances in Physics, vol. 56, pp. 167-242, 2007

[3] P.V. Fellman and R. Wright: Modeling terrorist networkscomplex systems at the mid-range, Complexity, ethics and creativity conference, vol. 18, 2003

[4] L.C. Freeman: Visualizing social networks, Journal of social structure, vol. 1, 2000

[5] G.H. Golub and C. Reinsch: Singular value decomposition and least squares solutions, Numerische Mathematik, vol. 14, pp. 403-420, 1970

[6] T.G. Kolda and D.P. O'Leary: A semidiscrete matrix decomposition for latent semantic indexing information retrieval, ACM Transactions on Information Systems (TOIS), vol. 16, 1998

[7] Y. Koren, R. Bell and C. Volinsky: Matrix factorization techniques for recommender systems, IEEE Computer, vol. 42, pp. 30-37, 2009

[8] V.E. Krebs: Uncloaking terrorist networks, First Monday, vol. 7, 2002

[9] D. Lee, H. Seung : Learning the parts of objects by nonnegative matrix factorization, Nature, vol. 401, pp. 788-791, 1999

[10] D. Liben-Nowell and J. Kleinberg: The link-prediction problem for social networks, Journal of the American Society for Information Science and Technology, vol. 58, pp. 1019-1031, 2007

[11] M. R. Czinkota, G. A. Knight, P. W. Liesch, J. Steen, Positioning terrorism in management and marketing: Research propositions Journal of International Management, Volume 11, Issue 4, pp. 581-604, 2005 


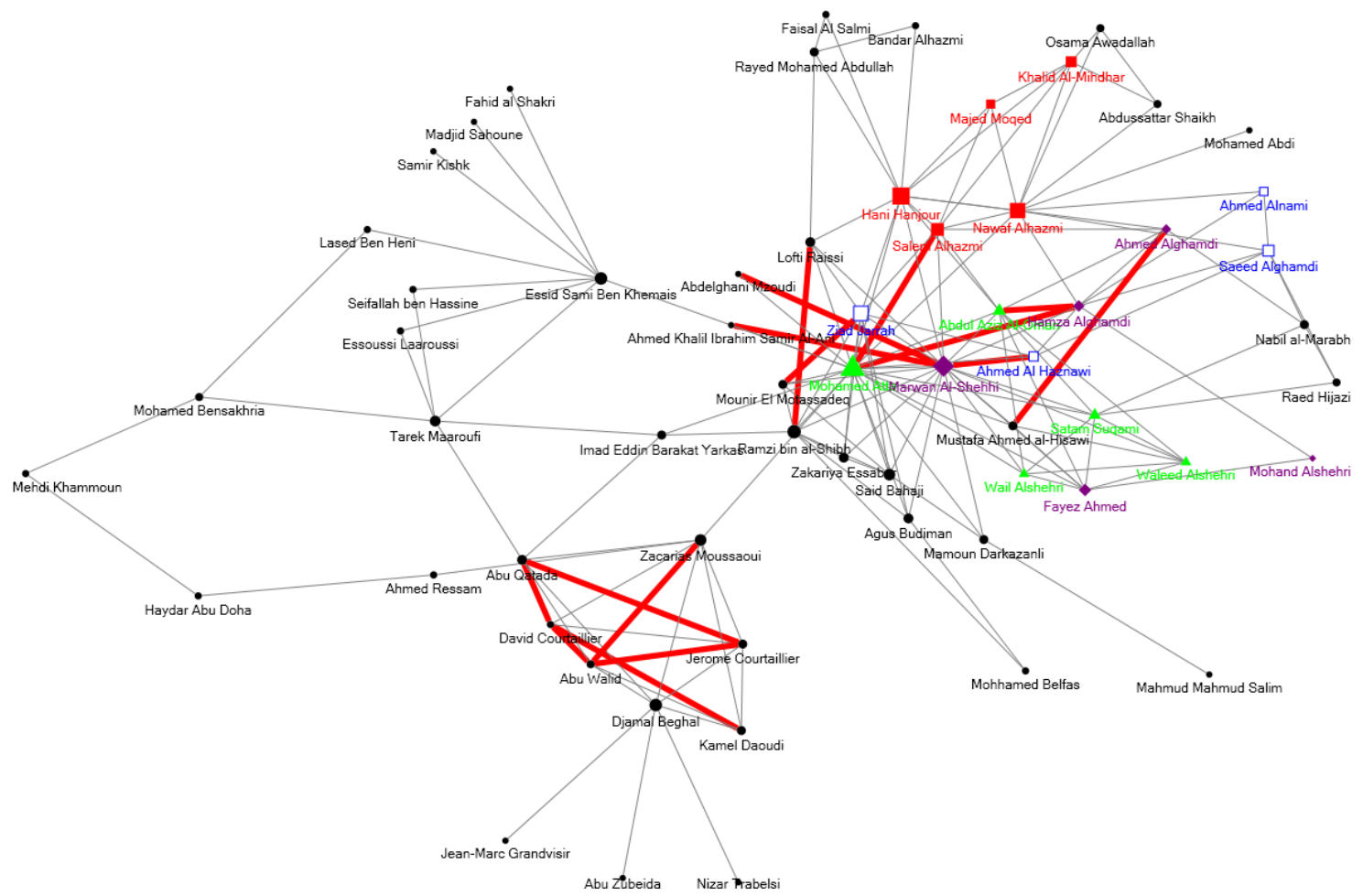

Figure 2. Terrorists network with highlighted link suggestions using SDD reduction (rank 10)

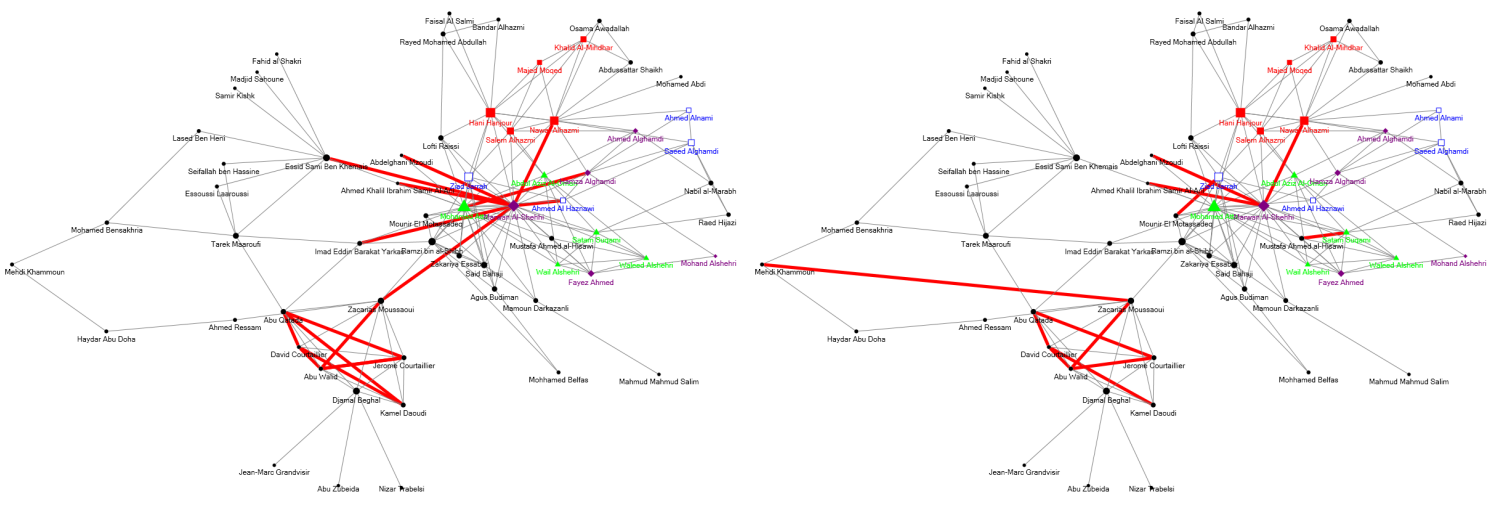

Figure 3. Terrorists network with highlighted link suggestions using SDD reduction (rank 5 and 20)

[12] W. T.H. Koh, Terrorism and its impact on economic growth and technological innovation Technological Forecasting and Social Change, Volume 74, Issue 2, pp. 129-138, 2007

[13] Edna F. Reid, Hsinchun Chen, Mapping the contemporary terrorism research domain International Journal of HumanComputer Studies, Volume 65, Issue 1, pp. 42-56, 2007

[14] Y. Wolf, O. Frankel, Terrorism: Toward an overarched account and prevention with a special reference to pendulum interplay between both parties Aggression and Violent Behavior, Volume 12, Issue 3, pp. 259-279, 2007

[15] A. Abadie, J. Gardeazabal, Terrorism and the world economy
European Economic Review, Volume 52, Issue 1, pp. 1-27, 2008

[16] C. Kollias, P. Messis, N. Mylonidis, S. Paleologou, Terrorism and the effectiveness of security spending in Greece: Policy implications of some empirical findings Journal of Policy Modeling, Volume 31, Issue 5, pp. 788-802, 2009

[17] A. Paraskevas, B. Arendell, A strategic framework for terrorism prevention and mitigation in tourism destinations Tourism Management, Volume 28, Issue 6, pp. 1560-1573, 2007

[18] B. S. Frey, How can business cope with terrorism? Journal of Policy Modeling, Volume 31, Issue 5, pp. 779-787, 2009 